\title{
Square wells, quantum wells and ultra-thin metallic films
}

\author{
Victor Barsan \\ Department of Theoretical Physics \\ IFIN-HH, Magurele-Bucharest, Romania
}

June 26, 2021

\begin{abstract}
The eigenvalue equations for the energy of bound states of a particle in a square well are solved, and the exact solutions are obtained, as power series. Accurate analytical approximate solutions are also given. The application of these results in the physics of quantum wells are discussed,especially for ultra-thin metallic films, but also in the case of resonant cavities, heterojunction lasers, revivals and super-revivals.
\end{abstract}

\section{Introduction}

The present paper illustrates two interesting facts, namely: (1) some important nanophysical systems, for instance quantum wells, can be understood with elementary quantum mechanics [1; and (2) elementary quantum mechanics offers still unexplored domains, for instance the transcendental equations of eigenenergy. In fact, in almost all 'exactly solvable problems' of quantum mechanics, only the Schroedinger equation for the wave functions has an exact solution, but the transcendental equation for the eigenvalues can be solved only numerically or graphically. The attempt of finding analytical - exact or numerical - solutions for these equations is largely unexplored, even if it might demand sometimes quite elementary mathematics. So, 'there is plenty of room at the bottom' - to use Feynman's words in a different context. Inter alia, in this article we shall undertake such an exercise - to obtain analytical solutions for the eigenenergy of bound states of a particle in a finite square well. Of course, the issue of the bound states of a square well has a long history, being discussed in about 40 papers, in the last six decades, to say nothing about textbooks; for a review, see [2].

Until the mid '80s, the quantum square well was just a problem of elementary quantum mechanics, with applications in obtaining the energy levels of quasifree electrons moving on long molecules or just below the free surface of a metal [3], or in the elementary theory of the Ramsauer-Townsed effect [4]. The odd 
parity states of the $1 \mathrm{D}$ square well are in the same time the $l=0$ solutions of the 3D problem of a particle moving in a 'radial square well', the so-called 'deuteron problem', see for instance [5], vol.1, Problem 63, p.162. A more subtle application refers to ultrathin conducting channels in field-effect transistors [6].

How this elementary problem became really important? The impressive progress of experimental physics in early ' 80 s made possible the fabrication of heterostructures and quantum wells [7, and the fact that the general principles of their physics can be easily understood, using the model of the "particle in a rectangular box" has been immediately exploited. The basic fact is that, in a very thin film, with planar surfaces, with mesoscopic or macroscopic longitudinal dimensions, and with nanoscopic transversal dimensions, the longitudinal momentum (or wave vector) of electrons is quantized according to cyclic boundary conditions, generating quasi-continuous values, and the transversal momentum (or wave vector) - according to rigid boundary conditions, generating quantized values; the transversal quantum number depends on the film thickness. The transversal motion of the electrons can be described as a 'quantum square well problem'. Consequently, the electron spectrum is split in subbands, indexed by the transversal quantum numbers, with a quasi-continuous energy given by quasi-continous longitudinal momentum. As the transversal momentum, which depends on film thickness, cannot be larger than the Fermi momentum, there is a connection between the film thickness and the Fermi momentum. Due to the fact that this connection involves discontinous and continous functions, via the transverse and longitudinal momenta, the physical quantities describing the system are characterized by functions with discontinous derivatives, or even with discontinuities. They produce an interesting physical behavior, namely quantum size effects (QSEs)

In the last two decades, the ultra-thin metallic films where subject of intense investigation, especially due just to such QSEs, observed in such systems, consisting of an oscillatory behavior of the film stability [8], of the lattice deformation [9], of the work function [10] etc., as a function of the number of atomic monolayers. The QSEs, predicted in the pioneering papers of Sandomirskii [1] and Schulte [12, are of considerable interest, from both practical and theoretical points of view. Metallic ultra-thin films are especially relevant for ferromagnetic materials, being responsible for the giant magnetoresistivity observed in the antiferromagnetic superlattice [13. Also, tuning the number of monolayers means, due to QSEs, tuning the work function, consequently tuning the chemical properties of the surface [10].

This quite rich physics can be explained using a quite elementary formalism, considering, as just mentioned, that the movement of electrons in a direction transversal to the surface of the metallic film is quantized using the quantum wells theory. Even the crude approximation of an infinite square well might be still useful, for instance for the calculation of the lattice deformation [9] or of the Fermi energy [14. These successes can be easily understood, due to the fact that, if the number of monolayers is not too small $(n \gtrsim 25)$, the role of deep levels is dominant, and the difference between the deep levels of a finite well and the corresponding levels (i.e. having the same quantum numbers) of the 
infinite well is negligible. However, if the number of monolayers is of the order of unity $(n \lesssim 5)$, it is more and more inappropriate to approximate the levels of the quantum wells, i.e. the levels of electrons moving normal to the surface, with the levels of an infinite well. This is why it is important to obtain the exact values of the bound states for a well of an arbitrary depth, or reasonably precise analytic approximations of these quantities.

The structure of this paper is the following. In Section 2, we expose the elementary problem of the finite square well, discussing carefully the eigenvalue problem; to find its solution means to obtain a set of functions, $\xi_{n}$ and $\zeta_{n}$,corresponding to the even and odd parity states. Section 3 is devoted to the so-called parabolic and cubic approximations for these functions. In Section 4, we put the transcendental equation for the energy eigenvalue in a differential form, and obtain an exact series expansion for $\xi_{n}$ and $\zeta_{n}$. The 3rd order approximation of this series - the Barker's approximation [15] - is discussed in the light of the exact and approximate solutions, as it is already used in several applications. In Section 5 we discuss the applications of our results, in three domains: ultra-thin metallic films, revivals and super-revivals, and electromagnetism. Several concluding remarks are presented in Section 6 .

\section{The quantum square well}

In this section, we shall present the quantum mechanical problem of a particle in a finite square well. There are in principle two ways of defining the potential of the well, namely taking the top of the well at $E=0$, or taking the bottom at $E=0$. In the first case, the advantage is that the bound states (situated "inside the well") have negative energy - the typical situation for bound states, in quantum mechanics. In the second case, the advantage is that, in the limit of a very deep well, the levels are close to the corresponding levels (having the same quantum number) of the infinite well. We shall examine in detail the first case, when the potential is:

$$
V(x)=-U \cdot \theta\left(\frac{a}{2}-|x|\right)
$$

and $\theta$ defines the Heaviside function. The second case is briefly discussed later on, see Eqs. (32), (33). The Schroedinger equation for a particle of mass $m$ moving in the potential (1) has the form:

$$
\left[-\frac{\hbar^{2}}{2 m} \frac{d^{2}}{d x^{2}}+V(x)-E\right] \psi(x)=0
$$

As the potential is invariant to space inversion, $V(x)=V(-x)$, the solutions have well-defined parity. If we introduce the wave vectors $k, k_{0}$ via the relations:

$$
E=-\frac{\hbar^{2}\left(k_{0}^{2}-k^{2}\right)}{2 m}, \quad U=\frac{\hbar^{2} k_{0}^{2}}{2 m}
$$


and the dimensionless quantity $P$, which characterizes both the particle and the potential:

$$
P=k_{0} a / 2=\sqrt{2 m U} \frac{a}{2 \hbar}, \quad p=\frac{1}{P}
$$

Also,

$$
\frac{U}{P^{2}}=2 \frac{\hbar^{2}}{m a^{2}}
$$

With this notation, the energy of the particle takes the form:

$$
E=-U\left[1-\left(\frac{k a}{2 P}\right)^{2}\right]
$$

The even solutions of (2) are:

$$
\begin{gathered}
\psi_{+}(x ; k)=A_{+}(k)\left[\theta\left(\frac{a}{2}-|x|\right) \cdot \cos k x+\right. \\
\left.+\theta\left(|x|-\frac{a}{2}\right) \cdot \cos k a \cdot \exp \left(\sqrt{k_{0}^{2}-k_{n}^{2}}(a / 2-x)\right)\right] \\
\psi_{+}(-x)=\psi_{+}(x)
\end{gathered}
$$

and the odd ones:

$$
\begin{gathered}
\psi_{-}(x ; k)=A_{-}(k)\left[\theta\left(\frac{a}{2}-|x|\right) \cdot \sin k x+\right. \\
\left.+\theta\left(|x|-\frac{a}{2}\right) \cdot \sin k a \cdot \exp \left(\sqrt{k_{0}^{2}-k_{n}^{2}}(a / 2-x)\right)\right] \\
\psi_{-}(-x)=-\psi_{-}(x)
\end{gathered}
$$

The explicit form of the normalization constants $A_{ \pm}(k)$ in $(7),(9)$ can be found for instance in [5] and will be not given here.

The energy eigenvalue equations can be conveniently written in terms of the wave vector $k$. For even states:

$$
\frac{\cos ^{2}(k a / 2)}{(k a / 2)^{2}}=\frac{1}{P^{2}}=p^{2}
$$

and for odd states:

$$
\frac{\sin ^{2}(k a / 2)}{(k a / 2)^{2}}=\frac{1}{P^{2}}=p^{2}
$$

With $k$ obtained from (11), (12), the eigenenergy is given by (6).

So, to solve the eigenvalue equations (11), (12) means to find the functions $\zeta(p), \xi(p)$ which satisfy the relations: 


$$
\frac{\sin \zeta(p)}{\zeta(p)}= \pm p, \quad \frac{\cos \xi(p)}{\xi(p)}= \pm p
$$

In order to clearly define the alternation of signs in (13), we shall identify the intervals of monotony of the functions $\sin x / x, \cos x / x$. Of course, the $x$ varaible used here and hereafter has nothing to do with the spatial coordinate, enterng in the Schroedinger equation (1) and in its solutions. The extremum points of the function $\cos x / x$ are given by the roots $r_{c n}$ of the equation:

$$
\tan x=-\frac{1}{x}
$$

where $r_{c n}$ is the root closest to $(n-1) \pi$ :

$$
r_{c n}=(n-1) \pi-\frac{(n-1) \pi}{(n-1)^{2} \pi^{2}-1}+\mathcal{O}\left(\frac{1}{n^{2}}\right)
$$

The extremum values of the function $\cos x / x$ are:

$$
M_{c n}=\frac{(-1)^{n+1}}{(n-1) \pi}+\mathcal{O}\left(\frac{1}{n^{2}}\right), \quad n>1
$$

It is easy to see that the eigenvalue equations for the even states are:

$$
\begin{gathered}
x \in\left(0, \frac{\pi}{2}\right): \frac{\cos x}{x}=p ; x \equiv \xi_{1}(p) \\
x \in\left(r_{c 2} \simeq \pi, \frac{3 \pi}{2}\right): \frac{\cos x}{x}=-p ; x \equiv \xi_{2}(p) \\
x \in\left(r_{c 3} \simeq 2 \pi, \frac{5 \pi}{2}\right): \frac{\cos x}{x}=p ; x \equiv \xi_{3}(p)
\end{gathered}
$$

and so on.

Similarly, the extremum points of the function $\sin x / x$ are located in the roots $r_{s n}$ of the equation:

$$
\tan x=x
$$

where $r_{s n}$ is the root closest to $\left(n-\frac{1}{2}\right) \pi$. The analogous of (15) is:

$$
r_{s n}=\left(n-\frac{1}{2}\right) \pi-\frac{1}{\left(n-\frac{1}{2}\right) \pi}+\mathcal{O}\left(\frac{1}{n^{2}}\right)
$$

The extremum values of the function $\sin x / x$ are:

$$
M_{s 1}=1 ; M_{s n}=\frac{(-1)^{n-1}}{\left(n-\frac{1}{2}\right) \pi}+\mathcal{O}\left(\frac{1}{n^{2}}\right), n>1
$$

The eigenvalue equations for odd states are: 


$$
\begin{gathered}
x \in(0, \pi): \frac{\sin x}{x}=p ; x \equiv \zeta_{1}(p) \\
x \in\left(r_{s, 2} \simeq \frac{3}{2} \pi, 2 \pi\right): \frac{\sin x}{x}=-p ; x \equiv \zeta_{2}(p) \\
x \in\left(r_{s, 3} \simeq \frac{5}{2} \pi, 3 \pi\right): \frac{\sin x}{x}=p ; x \equiv \zeta_{3}(p)
\end{gathered}
$$

and so on. Each of the Eqs. (17) - (19), (23) - (25) has a unique solution, $\xi_{1}(p), \xi_{2}(p), \xi_{3}(p)$, respectively $\zeta_{1}(p), \zeta_{2}(p), \zeta_{3}(p)$, for sufficiently small values of $p$. On the aforementioned intervals, the functions $\cos x / x, \sin x / x$ are monotonous, and for their restriction on each of these intervals, an inverse function exists. These inverse functions are, in the aforementioned cases, $\xi_{1}(p), \xi_{2}(p), \xi_{3}(p)$, respectively $\zeta_{1}(p), \zeta_{2}(p), \zeta_{3}(p)$. Their plots can be obtained as symmetric of the restrictions of the functions $\cos x / x, \sin x / x$ on their intervals of monotony, given by Eqs. (17) - (19), (23) - (25).

Graphically, the functions $\zeta(p), \xi(p)$ correspond to the intersections of the $x$-coordinates of the plots of the functions $\sin x / x, \cos x / x$ with the line $y=$ $\pm p$, on well defined intervals. The number of solutions depends on the value of $p$. There is always at least one solution $\xi(p)$ for any value of $p$, but the solutions $\zeta(p)$ exist only for $p<1$. In Fig. 1 , the plots of the functions $\sin x / x$ (plain), $\cos x / x$ (dashed) and $y= \pm p$, with $p=0.1$, are given. The $x$-coordinate of the intersection point of $\sin x / x$, with $y=p$, for $0<x<\pi$, defines the function $\zeta_{1}(p)$; with $y=-p$, for $r_{s, 2} \simeq \frac{3}{2} \pi<x<2 \pi$, the function $\zeta_{2}(p)$; with $y=p$, for $r_{s, 3} \simeq \frac{5}{2} \pi<x<3 \pi$, the function $\zeta_{3}(p)$, etc. Similarly, the $x$-coordinate of the intersection point of $\cos x / x$, with $y=p$, for $0<x<\pi / 2$, defines the function $\xi_{1}(p)$; with $y=-p$, for $r_{c 2} \simeq \pi<x<\frac{3 \pi}{2}$, the function $\xi_{2}(p)$ etc.

As already mentioned, a finite well only supports a fixed number of bound states. According to several authors (see for instance [16]), this number is given by:

$$
n_{\max }=\operatorname{int}\left(\frac{P}{\pi / 2}\right)+1
$$

In other words, if we define:

$$
p(N)=\frac{2}{(N-1) \pi}
$$

for $p=p(N)-0^{+}$, the well supports $N-1$ bound states, and for $p=p(N)+0^{+}$, it supports $N$ bound states. This statement is correct in the approximation

$$
r_{c n}=(n-1) \pi, \quad r_{s n}=\left(n-\frac{1}{2}\right) \pi
$$

i.e. when we identify the extremum points of the functions $\cos x / x, \sin x / x$ with those of the functions $\cos x, \sin x$. Rigorously speaking, the situation is 
slightly more complicated, in the following sense. If $p$ decreases from $\left|M_{c n}\right|+0^{+}$ to $\left|M_{c n}\right|-0^{+}$, a new root, namely $\xi_{n}$ shows up, and this corresponds to the $(2 n-1)$-th bound state. Similarly, if $p$ decreases from $\left|M_{s n}\right|+0^{+}$to $\left|M_{s n}\right|-0^{+}$, a new root, namely $\zeta_{n}$ shows up, and this corresponds to the $2 n$-th bound state.

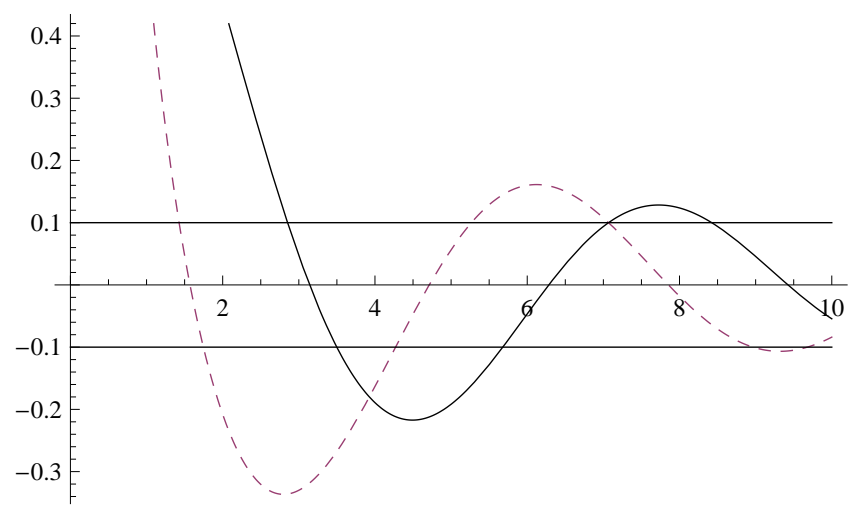

Figure 1: The $x$-coordonate of the intersection of the functions $\sin x / x$ (solid) and $\cos x / x$ (dotted) with the lines $y=p$ and $y=-p$, according to Eqs. (23) $(25)$ and $(17)-(19)$, defines the functions $\zeta_{n}(p)$ and $\xi_{n}(p)$, respectively.

The $n$-dependence of the coordinates of extremum points, $r_{c n}, r_{s n}$, has been obtained by approximating the tan function in (14), (21) with the first term of its Taylor series expansion. An alternative way of finding analytic approximations for the roots $r_{c n}, r_{s n}$ is to use the algebraic approximation for $\tan x$, indicated in [17. Of course, this is only an example of the usefulness of this algebraic approximation formula, which can be also used, for instance, to solve the eigenvalue equation for a particle moving in an infinite rectangular well, with a $\delta$-function barrier in the middle; indeed, this equation has the form $\tan z=-z / z_{0}[17$.

According to Eq. (6), the eigenvalues of the energy are:

$$
E_{n}=-U+U\left(\frac{k_{n} a}{2 P}\right)^{2}, n \geqslant 1
$$

If we consider a particle which is moving not in the potential $V(x)$ given by (1), but in a potential

$$
V^{(1)}(x)=V(x)+U
$$

then the energy levels will be given by:

$$
E_{n}^{(1)}=U\left(\frac{k_{n} a}{2 P}\right)^{2}=2 \frac{\hbar^{2}}{m a^{2}}\left(\frac{k_{n} a}{2}\right)^{2}, n \geqslant 1
$$


In correspondence to the parity of $n, k_{n} a / 2$ corresponds to the functions $\xi$ or $\zeta$, for instance $k_{1} a / 2=\xi_{1}(p), k_{2} a / 2=\zeta_{1}(p)$, etc. In general,

$$
E_{2 n-1}^{(1)}=2 \frac{\hbar^{2}}{m a^{2}} \xi_{n}^{2}, \quad E_{2 n}^{(1)}=2 \frac{\hbar^{2}}{m a^{2}} \zeta_{n}^{2}, \quad n \geqslant 1
$$

Let us remind that the quantity $k_{0}$ defined in (3) cannot be obtained putting $n=0$ in (29), (31), (32).

So, the eigenfunctions for the even states are:

$$
\psi_{2 n-1}(x)=\psi_{+}\left(x ; k_{2 n-1}\right)=\psi_{+}\left(x ; \frac{2}{a} \xi_{n}\right), \quad n \geqslant 1
$$

and for the odd states:

$$
\psi_{2 n}(x)=\psi_{-}\left(x ; k_{2 n}\right)=\psi_{+}\left(x ; \frac{2}{a} \zeta_{n}\right), n \geqslant 1
$$

As it has been already mentioned, the advantage of using the potential (1) consists in the fact that the energies of the particle "inside the well", i.e. the energy of the bound states, are negative, which corresponds to the usual convention adopted in quantum mechanics. However, the form (30) of the potential has the advantage - also mentioned previously - that its levels become, in the limit of a very deep well, the levels of the infinite well. It is indeed easy to see that, for $n \rightarrow \infty$, so for very deep wells, the quantization condition for the wave vector becomes $k_{n} a \simeq n \pi$, so

$$
k_{n} \simeq \frac{n \pi}{a}
$$

and the Eq. (31) becomes the equation of the energy levels of an infinite well:

$$
E_{n}^{(\infty)}=\frac{\pi^{2} \hbar^{2}}{2 m a^{2}} n^{2}
$$

\section{The parabolic and cubic approximations}

The parabolic and cubic approximations consist in approximating the restrictions of the functions $\cos x / x, \sin x / x$ on their monotony intervals, defined in Section 2, with segments of parabolas, or of cubic polynomials, having the same roots, extremum points and (in the case of cubic polynomials) slope in the root, as the exact functions. The detailed methods for obtaining these approximations have been indicated in [18, 2]. We shall give here only the results. The parabolic approximation for the functions $\xi, \zeta$ is:

$$
\xi_{n}^{(i p)}(x)=r_{c n}+\left(\left(n-\frac{1}{2}\right) \pi-r_{c n}\right) \sqrt{1-\frac{x}{M_{c n}}}, n>1
$$

Also, 


$$
\zeta_{n}^{(i p)}(x)=r_{s n}+\left(n \pi-r_{s n}\right) \sqrt{1-\frac{x}{M_{s n}}}, n>1
$$

They are defined on the intervals $\left(M_{\alpha n}, 0\right)$ for $n=$ even and on $\left(0, M_{\alpha n}\right)$, for $n=o d d$, with $\alpha=c$ for $\xi$ functions and $\alpha=s$ for $\zeta$ functions.

If the extremum points of the functions $\cos x / x, \sin x / x$ are approximated with the extremum points of the functions $\cos x, \sin x$, the formulas (37), (38) become:

$$
\begin{gathered}
\xi_{n}^{(s p)}(x)=(n-1) \pi+\sqrt{\frac{\pi^{2}}{4}-\frac{x}{4}(n-1) \pi^{3}} \\
\zeta_{n}^{(s p)}(x)=\left(n-\frac{1}{2}\right) \pi+\sqrt{\frac{\pi^{2}}{4}+\frac{x}{4}\left(n-\frac{1}{2}\right) \pi^{3}}
\end{gathered}
$$

and their intervals of definition change accordingly. As the formulas (39), (40) are sometimes referred to as 'simple parabolic approximation', we shall refer to (37), (38) as 'improved parabolic approximation'. This convention explains the upper indices in the definition of $\xi_{n}$ and $\zeta_{n}$ functions, Eqs. (37), (38) and (39), (40).

In order to introduce the cubic approximations for the functions $\xi$, $\zeta$, we have to define the quantities:

$$
X_{s n, M}=r_{s, n}-n \pi, \quad a_{s n, 1}=\frac{(-1)^{n}}{n \pi}
$$

and

$$
a_{s n, 3}=-\frac{2 M_{s n}}{X_{s n, M}^{3}}+\frac{a_{s n, 1}}{X_{s n, M}^{2}}, \quad a_{s n, 2}=\frac{3 M_{s n}}{X_{s n, M}^{2}}-\frac{2 a_{s n, 1}}{X_{s n, M}}
$$

Using the notations:

$$
A_{s n, 1}=\frac{a_{s n, 1}}{a_{s n, 3}}-\frac{1}{3} \frac{a_{s n, 2}^{2}}{a_{s n, 3}^{2}}<0 ; \quad A_{s n, 0}(x)=\frac{2}{27} \frac{a_{s n, 2}^{3}}{a_{s n, 3}^{3}}-\frac{1}{3} \frac{a_{s n, 1} a_{s n, 2}}{a_{s n, 3}^{2}}-\frac{x}{a_{s n, 3}}
$$

the cubic approximation for $\zeta_{n}(x)$ is:

$$
\zeta_{n}^{(c)}(x)=n \pi-\frac{a_{s n, 2}}{3 a_{s n, 3}}+\frac{2\left|A_{s n, 1}\right|^{1 / 2}}{\sqrt{3}} \sin \left(\frac{1}{3} \arcsin \left(\frac{\sqrt{27}}{2} \frac{A_{s n, 0}(x)}{\left|A_{s n, 1}\right|^{3 / 2}}\right)\right)
$$

Similarly, defining:

$$
X_{c n}=x-\left(n-\frac{1}{2}\right) \pi, \quad a_{c n, 1}=\frac{(-1)^{n}}{\left(n-\frac{1}{2}\right) \pi}
$$


and simply replacing the index $s$ by $c$ in (42), (43), we get the cubic approximation for $\xi_{n}(x)$.

$\xi_{n}^{(c)}(x)=\left(n-\frac{1}{2}\right) \pi-\frac{a_{c n, 2}}{3 a_{c n, 3}}+\frac{2}{\sqrt{3}}\left|A_{c n, 1}\right|^{1 / 2} \sin \left(\frac{1}{3} \arcsin \left(\frac{\sqrt{27}}{2} \frac{A_{c n, 0}(x)}{\left|A_{c n, 1}\right|^{3 / 2}}\right)\right)$

The cubic approximations are defined on the same intervals as the corresponding parabolic approximations.

As $\cos x / x$, for $0<x<\pi / 2$ cannot be approximated by a polynomial, the root $\xi_{1}$ cannot be obtained from the formulas (37) or (46). The function $\sin x / x$ for $0<x<\pi$ can be approximated by a polynomial, but cannot be obtained from the general formulas, as the interval of monotony is not of the form used for the evaluation of $\zeta_{n}$, with $n>1$. However, the same approach can be used, giving:

$$
\zeta_{1}^{(p)}(x)=\pi \sqrt{1-x}
$$

for the parabolic approximation (in this case, the simple and the improved approximation coincide), and

$$
\begin{gathered}
\zeta_{1}^{(c)}(x)=\frac{\pi(1-x)^{1 / 2}}{2^{1 / 2}} . \\
\cdot \frac{1}{-\frac{1}{\sqrt{3}} \sin \left(\frac{1}{3} \arcsin \left(\frac{3^{3 / 2}}{2^{5 / 2}}(1-x)^{1 / 2}\right)\right)+\cos \left(\frac{1}{3} \arcsin \left(\frac{3^{3 / 2}}{2^{5 / 2}}(1-x)^{1 / 2}\right)\right)}
\end{gathered}
$$

for the cubic approximation.

Some caution is needed while switching from $x$ to $p$ dependence in these formulas. In fact, the parabolic and cubic approximations have been obtained in a pure geometric context, and the functions $\xi_{n}(p), \zeta_{n}(p)$ in $(29),(32)$ - in a dynamic context, so, in order to use these approximations in the eigenvalue expressions, we have to write $\xi_{n}^{(c)}(p) \equiv \xi_{n}^{(c)}(|x| \rightarrow p), \zeta_{n}^{(c)}(p) \equiv \zeta_{n}^{(c)}(|x| \rightarrow p)$. The expressions of the cubic approximations for the functions indexed by $n=$ $2 \ldots 4$ are given in the Appendix.

\section{The differential form of the transcendental equa- tions}

We shall expose now a method of solving the eigenvalue equations, discovered and re-discovered several times, which generates an exact solution, in the form of a series expansion. As we shall see, the method works for a large class of transcendental equations. 
Let us take the derivatives in both sides of the equations (17)-(19) and (23)(25), putting:

$$
X_{2 n}(p)=\zeta_{n}(p), X_{2 n-1}(p)=\xi_{n}(p), n=1,2, \ldots,
$$

replacing $p$ by $x$ and relaxing the restriction $p>0$. The equations for the energy eigenvalues can be written in a unitary form:

$$
\frac{d X_{n}(x)}{d x}=-\frac{X_{n}(x)}{\sqrt{1-x^{2} X_{n}(x)^{2}}+x}
$$

with the initial condition:

$$
X_{n}(0)=\frac{n \pi}{2}
$$

It is remarkable that both the even, and the odd solutions, satisfy identical differential equations. The formula (50) allows us to obtain the derivatives of any order of $X_{n}(x)$ in an arbitrary point $x_{0}$ and, consequently, to construct the Taylor series for this function, with arbitrary precision. Choosing $x_{0}=0$, and putting:

$$
\frac{X_{n}(x)}{X_{n}(0)}=\frac{X_{n}(x)}{n \pi / 2}=x_{n}(x)=\sum_{m=0}^{\infty} q_{m}\left(\frac{n \pi}{2}\right) x^{m}
$$

we get the following exact series expansion for $x_{n}(x)$ :

$$
x_{n}(x)=\sum_{m=0}^{\infty} q_{m}\left(\frac{n \pi}{2}\right) x^{m}
$$

The first 6 functions $q_{m}$ are the following polynomials in the variable

$$
\frac{n \pi}{2} \equiv b_{n},
$$

namely

$$
\begin{gathered}
q_{0}\left(b_{n}\right)=1, \quad q_{1}\left(b_{n}\right)=-1, \quad q_{2}\left(b_{n}\right)=1 \\
q_{3}\left(b_{n}\right)=-\left(1+\frac{b_{n}^{2}}{6}\right), \quad q_{4}\left(b_{n}\right)=\left(1+\frac{2 b_{n}^{2}}{3}\right) \\
q_{5}\left(b_{n}\right)=-\left(1+\frac{5}{3} b_{n}^{2}+\frac{3}{2^{3} \cdot 5} b_{n}^{4}\right), \quad q_{6}\left(b_{n}\right)=\left(1+\frac{2 \cdot 5}{3} b_{n}^{2}+\frac{2^{3}}{3 \cdot 5} b_{n}^{4}\right)
\end{gathered}
$$

The polynomials $q_{2 n-1}(b), q_{2 n}(b)$ have the same order, $2 n-1$. We calculated quite a lot of such polynomials (see the Appendix) with the expectation that this exercise could help to guess a general formula for its coefficients. However, the presence of some large prime numbers in the products which enter in the 
coefficient of polynomials $q_{m}$, specially for odd indices, is somewhat discouraging for the attempt of finding general expressions for this quantities.

The limit $p \rightarrow 0$ corresponds to the infinite well. It is easy to see, using (35) and (53), that:

$$
E_{n}^{(1)}(p)=E_{n}^{(\infty)} \cdot x_{n}^{2}(x)
$$

with

$$
E_{n}^{(\infty)}=2 \frac{\hbar^{2}}{m a^{2}} X_{n}^{2}(0)
$$

Also, the energy levels of the semi-infinite well, corresponding to the limit $U_{0} \rightarrow \infty$ in (30), are given by:

$$
E_{n}^{(s \infty)}=\frac{2 \hbar^{2} X_{2 n-1}(0)^{2}}{m a^{2}}=\frac{2 \hbar^{2} \pi^{2}}{m a^{2}}\left(n-\frac{1}{2}\right)^{2}
$$

Numerical studies show that the series expansion (53) can be used only in a small interval near the origin; its value in another point must be obtained constructing the series expansion near that point. This is why, for practical calculations, it is convenient to use various approximations of the exact formula.

Restricting the series (53) at its first 3 terms, we get Barker's approximation [15]:

$$
X_{n}^{(B)}(p)=\frac{n \pi}{2}\left(1-p+p^{2}-\left(1+\frac{1}{6}\left(\frac{n \pi}{2}\right)^{2} p^{3}\right)\right)
$$

Barker's approximation has been used in several applications of the square well, for instance in the study of revivals and super-revivals. As given by its author, the approximation formula is:

$$
\begin{gathered}
\alpha_{n}(p)=\frac{1}{1+p} \frac{n \pi}{2}-\frac{p^{3}}{6(1+p)^{6}}\left(\frac{n \pi}{2}\right)^{3}= \\
=\frac{1}{2} \pi n-\frac{1}{2} \pi n p+\frac{1}{2} \pi n p^{2}-\frac{1}{2} \pi n\left(1+\frac{1}{24} \pi^{2} n^{2}\right) p^{3}+\ldots
\end{gathered}
$$

With our notations,

$$
\alpha_{n}(p)=X_{n}^{(B)}(p)
$$

As Barker's approximation is a consequence of the cubic approximation of the sin function, its precision goes up to the third order terms, so the terms not explicitly written in the r.h.s. of (62) must be omitted. In other words, the correct form of the "Barker's approximation" is the polynomial form given in (61), not the fractional form given in the original paper. We can check this property verifying that 


$$
\frac{\sin \left(\zeta_{1}^{(B)}(p)\right)}{\zeta_{1}^{(B)}(p)}=p+\mathcal{O}\left(p^{4}\right), \frac{\sin \left(\zeta_{2}^{(B)}(p)\right)}{\zeta_{2}^{(B)}(p)}=-p+\mathcal{O}\left(p^{4}\right)
$$

and so on. Such a property has any polynomial approximation of the exact solution. For instance, putting:

$$
\zeta_{n}^{(N)}(p)=X_{2 n}^{(N)}(p)=n \pi \sum_{m=0}^{N} q_{m}(n \pi) p^{m}
$$

we get:

$$
\frac{\sin \left(\zeta_{n}^{(N)}(p)\right)}{\zeta_{n}^{(N)}(p)}=(-1)^{n+1} p+\mathcal{O}\left(p^{N+1}\right)
$$

Graphical and numerical considerations show that Barker's approximation cannot be used for $\xi_{1}$ and $\zeta_{1}$, but is useful for any other case, being more precise for smaller values of $p$. The parabolic approximation can be used, qualitatively, for $\zeta_{1}$, but is less precise than Barker's one, in all other cases. The cubic approximation gives excellent results in all cases, excepting $\xi_{1}$, where de Alcantara Bonfim - Griffith solution should be used. Polynomial approximations of the exact solutions, higher than Barker's, are useful only for small arguments, so Barker's approximation can be considered as the most convenient polynomial approximation. For the illustration of these conclusions, we give here the plots for the $\zeta_{1}$ and $\zeta_{2}$ functions.

In Fig. 2, we can see how accurately the solutions $\zeta_{1}^{(B)}, \zeta_{1}^{(s p)}$ and $\zeta_{1}^{(c)}$ satisfy the equation (23). The functions

$$
\frac{\sin \left(\zeta_{1}^{(B)}(p)\right)}{\zeta_{1}^{(B)}(p)}, \frac{\sin \left(\zeta_{1}^{(s p)}(p)\right)}{\zeta_{1}^{(s p)}(p)}, \frac{\sin \left(\zeta_{1}^{(c)}(p)\right)}{\zeta_{1}^{(c)}(p)}
$$

are plotted with solid black, solid green and dotted black lines, respectively; the function $f_{1}(p)=p$ - with red. Barker's approximation is unusable, the parabolic one - quite poor, and the cubic one - very good. In Fig. 3, we check, similarly, how accurately the solutions $\zeta_{2}^{(B)}, \zeta_{2}^{(s p)}$ and $\zeta_{2}^{(c)}$ satisfy the equation (24); the conventions are similar, excepting the fact that instead of the function $f_{1}$, the function $f_{2}(p)=-p$ is plotted. Now, the Barker approximation is reasonably correct, especially for small values of the argument, the parabolic one is poorer than Barker's, and the cubic one is very accurate. These conclusions remain valid for the higher orders of both $\zeta$ and $\xi$ functions, with the remark that, in general, Barker's approximations becomes quite poor for large values of the argument.

Maybe because the rectangular well is, from the point of view of advanced physics, an elementary problem (even if, from the mathematical point of view, the eigenvalue equations for the energy are not at all trivial), it is sometimes 


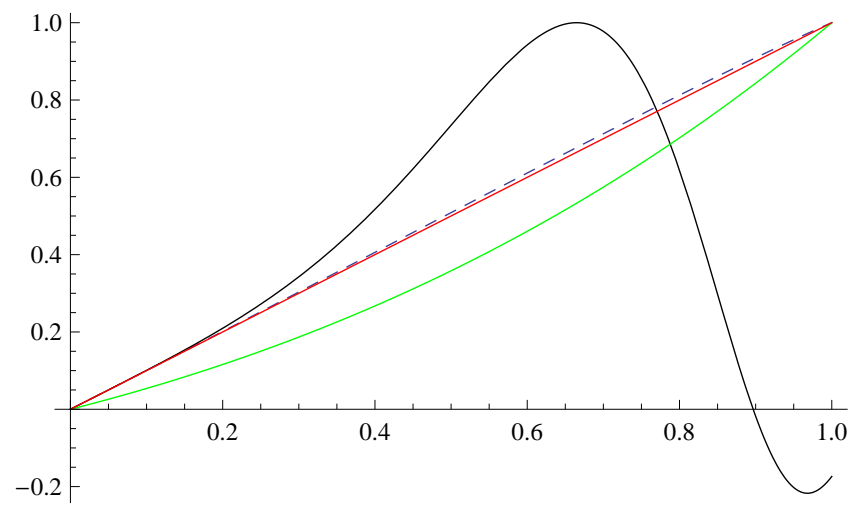

Figure 2: The functions $\frac{\sin \left(\zeta_{1}^{(B)}(p)\right)}{\zeta_{1}^{(B)}(p)}$ (solid, black), $\frac{\sin \left(\zeta_{1}^{(s p)}(p)\right)}{\zeta_{1}^{(s p)}(p)}$ (solid, green), $\frac{\sin \left(\zeta_{1}^{(c)}(p)\right)}{\zeta_{1}^{(c)}(p)}$ (dotted, black) and $f_{1}(p)=p$ (solid, red).

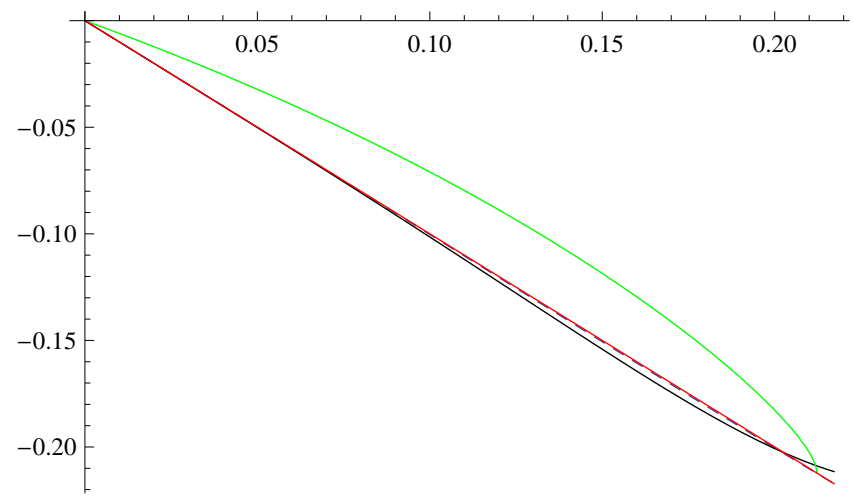

Figure 3: The functions $\frac{\sin \left(\zeta_{2}^{(B)}(p)\right)}{\zeta_{2}^{(B)}(p)}$ (solid, black), $\frac{\sin \left(\zeta_{2}^{(s p)}(p)\right)}{\zeta_{2}^{(s p)}(p)}$ (solid, green $), \frac{\sin \left(\zeta_{2}^{(c)}(p)\right)}{\zeta_{2}^{(c)}(p)}$ (dotted, black) and $f_{2}(p)=-p$ (solid, red). 
underestimated, and several results obtained by various authors remained unnoticed, and re-obtained in other forms; so, the domain has many repetitions and overlappings. For instance, in [19], the authors use the same method as that just exposed here in order to find a series expansion for the solutions of the eigenvalue equations, but the expansion used by them, equivalent to (65), is limited to second order terms. In fact, the precision of their research, which is focused on the physical properties of a mesoscopic film of thickness $L_{z}$, does not request a better precision. However, this precision is inferior to the precision of the Barker approximation which, as we could see, goes to third order terms. Apparently, it seems that in [19] Barker's results are ignored, and re-obtain the series using a different method.

Also, in spite of the fact that the equivalence of the Sturm - Liouville problems for electromagnetic waves and wave functions is well known since the early years of the quantum mechanics, the results obtained in the square well problem seem to remain unused by the researchers studying light propagation in waveguides or in other simple geometries. Reciprocally, the very detailed analysis of the eigenvalue equations, very similar to that of a square well, used in the propagation of electromagnetic waves [20, where a number of approximate solutions have been proposed (see for instance the references [90, 92] in [20]), seem to have remained unnoticed by people working in quantum mechanics.

\section{Applications: ultrathin metallic films, revivals and electromagnetism}

\subsection{Ultra-thin metallic films}

Even if the infinite square well is a very crude model, it can explain satisfactorily many aspects of the physics of metallic thin films. This is not very surprising, as the energy levels of a deep square well are acceptably approximated, for large quantum numbers, by the levels of an infinite well. The approximation fails for the first ones $(n \lesssim 5)$, but for a film with $n \gtrsim 25$ monolayers, the effect is not dramatic. The infinite well model has been improved, taking into account, in an approximative manner, the fact that the electron moving in the well can penetrate its walls; this is the so-called phase accumulation model (PAM). The model is exposed in a number of papers, 21], 22], 13]; concerning PAM for a metal - semiconductor interface, see [23]. However, even PAM gives very inaccurate results for very thin films. The fact that both the infinite well model and PAM are inappropriate for shallow wells (for instance, in an $1 \mathrm{ML} \mathrm{Ag} \mathrm{film,} \mathrm{only}$ a single QW state exists [22]) is discussed by several researchers. For instance, in $[8$, the authors warn about the fact that we have to be very cautious when we apply the infinite well model to very thin films; in fact, they find that the stability of a thin film of less than 5 monolayers depends sensitively on the height of interface energy barriers, (see [ $]$, p.6). In the photoemission studies of QW states in thin films, reported in 24] and [25, the data for the first 3 monolayers 
are excluded from the fit, in a PAM analysis. The angle - resolved photoemission is indeed an important tool for the study of electron states in QWs. In fact, there is a close analogy between a standing electromagnetic wave propagating between two reflecting surfaces and an electron moving in a square potential well [26. The first case corresponds to the Fabry - Perot interferometer, which shows a set of peaks in optical transmission; the second, to an electron confined in QW, formed in an ultra-thin metallic film. Electron confinement within a film results in discrete QW states, observable by angle-resolved photoemission. For a such film of $\mathrm{Ag}$ on $\mathrm{Fe}$, angle-resolved photoemission spectra are well explained by PAM for number of ML ¿2, but fails for 1 or 2 monolayers. [27.

So, the exact treatment of the finite well, or analytic approximations of the exact solutions are necessary for a correct description of the shallow wells (presuming that the model itself is still usable in all ultra-thin films). Our results can be directly used in the approach of $\mathrm{Wu}$ and Zhang [8] who apply the finite well model to the study of very thin films, but use numerical instead of analytical solutions. For freestanding films, where the electrons move in a symmetric square well of barrier height

$$
V_{0}=W_{m}+E_{F}
$$

the theory works without any adjustable parameter. The freestanding films are of great theoretical interest, as they can serve as reference points to see how substrates affect film properties.

For a film on a semiconductor substrate, the film is modeled as electrons residing in an asymmetric well, so:

$$
V(x<|a| / 2)=V_{1}=W_{m 1}+E_{F}, \quad V(x>|a| / 2)=V_{2}=W_{m 2}+E_{F}
$$

As $W_{m 1}, W_{m 1}$ are comparable, $\left|V_{1}-V_{2}\right| \ll V_{1}, V_{2}$, and the eigenenergy can be obtained using the perturbation theory, considering the symmetric well as exactly solvable problem. The same remarks applies for an electron in a corrugated box, for instance if the corrugated periodic potential is ([ $[$, eq. (29)):

$$
V_{c}(z)=v \cos \frac{2 \pi z}{d_{0}}
$$

with $\nu \ll V_{0}$.

For solid-solid and solid-vacuum interfaces, the confinement potential is generally finite and rounded. Examples of such potentials, for multilayered nanostructures, obtained using the dynamical mean-field theory approach, can be found in [28, Sect. 3.6. If this more realistic form of the potential has to be taken into account, its eigenstates can be obtained by simple perturbation calculation, from the eigenstates of the rectangular well.

It is well known that the Sturm - Liouville problems in optics and quantum mechanics can be identical 29], 30, so the quantized values of the wave vector of the quantum mechanical problem correspond perfectly to the normal modes of transversal electric field in resonant cavities, wave guides or heterostructure 
lasers, so our solutions of the energy eigenvalues can be applied also in optics and electromagnetism.

\subsection{Revivals}

As it is well known, wave packets in infinite square wells produce revivals, and in finite square wells - both revivals and super-revivals. Analyzing numerically the superrevivals in finite square wells, 31 notices that the Barker's formula:

$$
E_{n} \simeq \frac{P^{2}}{(P+1)^{2}} \frac{\pi^{2} \hbar^{2}}{2 m L^{2}} n^{2}
$$

(actually, the first term of the Barker's formula, obtained in fact by Garrett [32]) is less precise for shallow wells, than for deep wells. This property clearly results from our analysis of exact and approximate solutions of the square well problem. The same approximation is used by [33. However, no analytic result can be obtained in this way, as the $n$-dependence of the eigenenergy is qualitatively different in the two cases (for the finite square well, the simple $n^{2}$ dependence disappears), and this difference is smeared out in the Barker approximation.

The solutions of the transcendental equations allows us to find the analytic expressions of the revivals and superrevivals times for the finite square well, according to the Eqs. (10), (26-28) of [16].

\subsection{Electromagnetism}

The electromagnetic wave propagation in the symmetric three-layer slab waveguide is an example of phenomena described by a Sturm - Liouville system essentially identical to that of the quantum square well. Due to the large number of quantities to be defined in order to follow in detail the similarity of the two phenomena, we shall omit a detailed presentation, mentioning only that the equations giving the propagation constant (the wave vector) of the even and odd transversal electric modes, and of the even and odd transversal magnetic modes, respectively $(2.4-45),(2.4-54)$; (2.4-60), (2.4-66) in [34] correspond exactly to the eigenvalue equations for the energy of even and odd bound states in a quantum square well, respectively Eqs. (25.4e) and (25.4o) of [5].

\section{Conclusions}

This paper represents an illustration of the situation when solutions of simple problems of quantum mechanics can be used for description of interesting phenomena in nanophysics. Specifically, we have referred to exact solutions of the eigenvalue equations for the energy of bound states of a particle in a rectangular well. Even if the physics of this problem is elementary, its mathematics is not trivial, and consists in solving a transcendental equation. Both exact and approximate solutions are obtained, with various degrees of accuracy. The results 
are interesting for the physics of quantum wells, mainly for the important case of ultra-thin metallic films. Besides the case of electrons in rectangular quantum wells, the case of rugose bottom wells can be treated, with an elementary perturbation theory. Also, the theory described here has applications in the domain of heterojunction lasers and in the electromagnetic wave propagation in three slab dielectric waveguides.

\section{A Appendix}

The function $\xi_{1}$ cannot be obtained using the polynomial approximations described in Section 3. Very accurate analytical approximations for $\xi_{1}$ (with an error of about $10^{-3}$ ) have been proposed by de Alcantara Bonfim and Griffith [17, using an algebraic approximations for the cos function.

The cubic approximation for the following $4 \xi_{n}$ functions is:

$$
\begin{array}{r}
\xi_{2}^{(c)}(p)=4.2409+2.8851 \sin \left(\frac{1}{3} \arcsin (0.4728-4.3767 p)\right) \\
\xi_{3}^{(c)}(p)=7.6132+2.9840 \sin \left(\frac{1}{3} \arcsin (0.2300-7.6906 p)\right) \\
\xi_{4}^{(c)}(p)=10.8580+3.0803 \sin \left(\frac{1}{3} \arcsin (0.1336-10.6235 p)\right) \\
\xi_{5}^{(c)}(p)=14.0607+3.1484 \sin \left(\frac{1}{3} \arcsin (0.0729-13.439 p)\right)
\end{array}
$$

The cubic approximation for $\zeta_{1}$ is given in Section 3. The next four $\zeta_{n}$ functions are:

$$
\begin{array}{r}
\zeta_{2}^{(c)}(p)=5.9562+2.9256 \sin \left(\frac{1}{3} \arcsin (0.3297-6.121 p)\right) \\
\zeta_{3}^{(c)}(p)=9.24337+3.03623 \sin \left(\frac{1}{3} \arcsin (0.17839-9.1793 p)\right) \\
\zeta_{4}^{(c)}(p)=12.4627+3.1173 \sin \left(\frac{1}{3} \arcsin (0.0996-12.0402 p)\right) \\
\zeta_{5}^{(c)}(p)=15.6911+3.2908 \sin \left(\frac{1}{3} \arcsin (0.01538-14.3186 p)\right)
\end{array}
$$


The coefficients $q_{7}, \ldots q_{16}$, of the exact series expansion (53)

$$
\begin{aligned}
& q_{7}(b)=-\left(1+\frac{5 \cdot 7}{2 \cdot 3} b^{2}+\frac{7 \cdot 37}{2^{3} \cdot 3 \cdot 5} b^{4}+\frac{5}{2^{7} \cdot 7} b^{6}\right) \\
& q_{8}(b)=\left(1+\frac{2^{2} \cdot 7}{3} b^{2}+\frac{2 \cdot 7^{2}}{3 \cdot 5} b^{4}+\frac{2^{4}}{5 \cdot 7} b^{6}\right) \\
& q_{9}(b)=-\left(1+2 \cdot 7 b^{2}+\frac{7 \cdot 47}{2^{2} \cdot 5} b^{4}+\frac{3229}{2^{2} \cdot 3^{2} \cdot 5 \cdot 7} b^{6}+\frac{5 \cdot 7}{2^{7} \cdot 3^{2}} b^{8}\right) \\
& q_{10}(b)=\left(1+2^{2} \cdot 5 b^{2}+\frac{2 \cdot 7 \cdot 13}{5} b^{4}+\frac{2^{4} \cdot 41}{3^{2} \cdot 7} b^{6}+\frac{2^{7}}{3^{2} \cdot 5 \cdot 7} b^{8}\right) \\
& q_{11}(b)=-\left(1+\frac{5 \cdot 11}{2} b^{2}+\frac{7 \cdot 11 \cdot 19}{2^{2} \cdot 5} b^{4}+\frac{11 \cdot 1571}{2^{3} \cdot 3^{2} \cdot 7} b^{6}+\frac{11 \cdot 59 \cdot 181}{2^{7} \cdot 3^{2} \cdot 5 \cdot 7} b^{8}+\frac{3^{2} \cdot 7}{2^{8} \cdot 11} b^{10}\right) \\
& q_{12}(b)=\left(1+\frac{2 \cdot 5 \cdot 11}{3} b^{2}+\frac{2 \cdot 11 \cdot 31}{5} b^{4}+\frac{2^{2} \cdot 11 \cdot 139}{3^{2} \cdot 7} b^{6}+\frac{2^{3} \cdot 11 \cdot 479}{3^{4} \cdot 5 \cdot 7} b^{8}+\frac{2^{8}}{3^{2} \cdot 7 \cdot 11} b^{10}\right) \\
& q_{13}(b)=-\left(1+\frac{11 \cdot 13}{3} b^{2}+\frac{11 \cdot 13 \cdot 67}{2^{5} \cdot 5} b^{4}+\frac{11 \cdot 13 \cdot 17 \cdot 127}{2^{2} \cdot 3^{2} \cdot 5 \cdot 7} b^{6}+\frac{11 \cdot 13 \cdot 23 \cdot 6679}{2^{7} \cdot 3^{4} \cdot 5 \cdot 7} b^{8}+\right. \\
& \left.+\frac{13 \cdot 211 \cdot 2609}{2^{7} \cdot 3^{2} \cdot 5^{2} \cdot 7 \cdot 11} b^{10}+\frac{3 \cdot 7 \cdot 11}{2^{10} \cdot 13} b^{12}\right) \\
& q_{14}(b)=\left(1+\frac{2 \cdot 7 \cdot 13}{3} b^{2}+\frac{2 \cdot 7 \cdot 11 \cdot 13}{5} b^{4}+\frac{2^{2} \cdot 11 \cdot 13 \cdot 311}{3^{2} \cdot 5 \cdot 7} b^{6}+\frac{2^{4} \cdot 11 \cdot 13 \cdot 37}{3^{4} \cdot 5} b^{8}+\right. \\
& \left.\frac{2^{6} \cdot 13 \cdot 59}{3^{2} \cdot 5^{2} \cdot 11} b^{10}+\frac{2^{10}}{3 \cdot 7 \cdot 11 \cdot 13} b^{12}\right) \\
& q_{15}(b)=-\left(1+\frac{5 \cdot 7 \cdot 13}{2 \cdot 3} b^{2}+\frac{7^{2} \cdot 11^{2} \cdot 13}{2^{3} \cdot 3 \cdot 5} b^{4}+\frac{11 \cdot 13 \cdot 8521}{2^{4} \cdot 3^{2} \cdot 7} b^{6}+\frac{11 \cdot 13 \cdot 79 \cdot 2917}{2^{7} \cdot 3^{4} \cdot 5} b^{8}+\right. \\
& \left.\frac{7 \cdot 13 \cdot 1206053}{2^{8} \cdot 3^{4} \cdot 5 \cdot 11} b^{10}+\frac{17911 \cdot 135721}{2^{10} \cdot 3^{3} \cdot 5^{2} \cdot 7 \cdot 11 \cdot 13} b^{12}+\frac{11 \cdot 13}{2^{11} \cdot 5} b^{14}\right)
\end{aligned}
$$




$$
\begin{aligned}
q_{16}(b)= & \left(1+\frac{2^{3} \cdot 5 \cdot 7}{3} b^{2}+\frac{2^{2} \cdot 7 \cdot 13 \cdot 41}{3 \cdot 5} b^{4}+\frac{2^{4} \cdot 11 \cdot 13 \cdot 67}{3^{2} \cdot 5 \cdot 7} b^{6}+\frac{2 \cdot 11 \cdot 13 \cdot 2473}{3^{4} \cdot 5} b^{8}+\right. \\
& \left.\frac{2^{5} \cdot 13 \cdot 4201}{3^{4} \cdot 5 \cdot 11} b^{10}+\frac{2^{6} \cdot 266681}{3^{3} \cdot 5^{2} \cdot 7 \cdot 11 \cdot 13} b^{12}+\frac{2^{11}}{3^{2} \cdot 5 \cdot 11 \cdot 13} b^{14}\right)
\end{aligned}
$$

Acknowledgement 1 The financial support of the ANCS project PN 0937 01 06, of the JINR Dubna - IFIN-HH Magurele-Bucharest project no. 01-31072-2009/2013 and of the CEI Cooperation Fund Project No. 1202.161-13 are kindly acknowledged.

\section{References}

[1] V. V. Mitin, D. I. Sementsov, N. Z. Vagidov: Quantum Mechanics for Nanostructures, Cambridge University Press (2010)

[2] V. Barsan, R. Dragomir, Optoel.Adv.Mat - Rapid Commun. 6, 917 (2012)

[3] W.A. Harrison: Applied Quantum Mechanics, World Scientific (2000)

[4] S. Van Wyk: Computer Solutions in Physics, World Scientific (2011)

[5] S. Fluegge: Practical Quantum Mechanics, Springer-Verlag Berlin Heidelberg New York (1971)

[6] T. Ando, A. B. Fowler, F. Stern, Rev.Mod.Phys. 54, 437 (1982)

[7] R. M. Kolbas, N. Holonyak, Jr.(1984), Amer.J.Phys. 52, 431 (1984)

[8] B. Wu, Z. Zhang, Phys.Rev.B77, 035410 (2008)

[9] P. Czosche et al., Phys.Rev.Lett. 91, 226801 (2003)

[10] J. Kim et al., Proc.Natl.Ac.Sci. 107, 12761 (2010)

[11] V.B. Sandomirskii, JETF 52, 158 (1967); Sov.Phys. JETP 25, 101 (1967)

[12] F.K. Schulte, Surf.Sci. 55, 427 (1976)

[13] Z. Q. Qiu, N. V. Smith: J.Phys.:Cond.Matter 14, R169 (2002)

[14] W. A. Atkinson, A. J. Slavin, Amer.J.Phys. 7443 (2008)

[15] B. I. Barker, G. H. Rayborn, J. W. Ioup, G. E. Ioup, Am.J.Phys. 59, 1038 (1991)

[16] D. L. Aronstein, C. R. Stroud, Amer.J.Phys. 68, 943 (2000) 
[17] O. F. de Alcantara Bonfim, D. J. Griffiths, Amer.J.Phys. 74, 43 (2006)

[18] V. Barsan, Rom.Rep.Phys. 64, 685 (2012)

[19] V. V. Pogosov, V. P. Kurbatsky, E. V. Vasyutin, Phys.Rev.B195410 (2005)

[20] D. Marcuse, Light transmission optics, Van Nostrand, New York (1982)

[21] N. V. Smith, PhysRev B32, 3549 (1985)

[22] M. Milun, P. Pervan, D.P. Woodruff, Rep.Prog.Phys. 65, 99 (2002)

[23] S.-J. Tang et al. Phys.Rev.Lett. 107, 066802 (2011)

[24] T.-C. Chiang, Photoemission studies of QW states in thin films, Surf.Sci.Rep.39, 181 (2000)] and [Chiang2000PRB] PhysRev B61 1804 (2000)

[25] T.-C. Chiang, Phys.Rev. B61, 1804 (2000)

[26] C. Cohen-Tannoundji, B. Diu, F. Laloe: Quantum Mechanics, Wiley, New York (1977)

[27] J. J. Paggel, T. Miller, T.-C. Chiang, Science 283, 1709 (1999)

[28] J. K. Freericks: Transport in multilayered nanostructures, Imperial College Press, 2006

[29] R. J. Black, A. Ankiewicz, Am.J.Phys.53, 554 (1985)

[30] V. Barsan: Waveguides, resonant cavities, optical fibers and their quantum counterparts, in: V. Barsan, R. P. Lungu (Eds.): Trends in electromagnetism, InTech, 2011

[31] A. Venugopalan, A. G. Agarwal, Phys.Rev.A59, 1413 (1999)

[32] S. Garrett, Amer.J.Phys. 47, 195 (1979)

[33] D. L. Aronstein, C. R. Stroud, Phys.Rev.A55, 4526 (1997)

[34] H. C. Casey, Jr., M. B. Panish: Heterostructure lasers, Academic Press, New York, San Francisco, London (1978) 